\title{
An Identification Scheme for Robot Actuator Faults*
}

\author{
Alessandro De Luca Raffaella Mattone \\ Dipartimento di Informatica e Sistemistica \\ Università di Roma 'La Sapienza' \\ Via Eudossiana 18, 00184 Roma, Italy \\ \{deluca,mattone\}@dis.uniromal.it
}

\begin{abstract}
We present a scheme for identifying the time profile of actuator faults that may affect a robot manipulator. Starting from our previous method for fault detection and isolation (FDI) based on generalized momenta, fault identification is additionally obtained through the $\mathcal{H}_{\infty}$-design of a state observer for uncertain systems. For each separate fault channel, the identifier consists of a linear filter driven by the corresponding residual signal. Under the weak assumption of bounded time derivative for the otherwise unknown fault input to be identified, the fault estimation error is shown to be ultimately uniformly bounded, with ultimate bound that can be set arbitrarily small. The information on the type and severity of the fault may then be used for reconfiguring the control strategy. Experimental results on a $2 R$ planar manipulator are presented.
\end{abstract}

Index Terms - Fault detection and isolation, fault identification, robot actuators, $\mathcal{H}_{\infty}$-based state observers.

\section{INTRODUCTION}

The ability to automatically recognize the occurrence and the nature of faults possibly affecting actuators or sensors of a robotic system is a prerequisite for the design of fault tolerant control strategies with active reconfiguration [1]. In the presence of a generic fault, three phases can be considered: detection, isolation, and identification [2]. Fault detection consists in the generation of a diagnostic signal (residual) triggered by a significant deviation of the robot from the expected behavior, based on the processing of available signals and/or the use of a dynamic model. Fault isolation is achieved when the failed component is individuated. Fault identification consists in estimating the time behavior of the fault, i.e., of the difference between expected and actual behavior of the variable affected by a fault (e.g., the measure provided by a sensor or the torque produced by an actuator). This information can be used to classify the occurred fault (e.g., an additive bias to a sensor measurement or a power loss of an actuator) and its severity, in order to choose the most appropriate fault recovery strategy.

Several schemes have been proposed for the detection of actuator faults in robot manipulators [3], [4], [5]. The most efficient ones, which provide also fault isolation without requiring acceleration measurements nor inversion of the robot inertia matrix, are based on filtered torques [6] or on the use of generalized momenta [7]. Both these model-based techniques admit adaptive versions, coping with structured uncertainty in the robot dynamics (see [8]).

\footnotetext{
${ }^{*}$ Work supported by the EU project EU-IST-2001-32122 IFATIS.
}

More recently, the problem of identifying actuator fault profiles has been addressed in [9], building upon the results in [6]. Under the assumption of bounded faults, with known bounded first and second time derivative, the estimation error is shown to converge asymptotically to zero (in the absence of disturbances). The scheme uses a robot velocity observer and involves the use of discontinuous signals and the inversion of the inertia matrix. On the other hand, the fault detection and isolation (FDI) method in [7] already provides residuals that are low-pass filtered versions of the original faults. Although the filter bandwidth could be set as large as desired in principle, practical limitations are imposed by input and measurement noise and by the uncertainties in the robot dynamic model.

Motivated by these results, we introduce here a different identification scheme based on a robust $\left(\mathcal{H}_{\infty}\right)$ observer design. In fact, the fault identification problem can be reformulated as that of designing a state observer for a linear system driven by the residuals and in the presence of unknown but bounded disturbances [10]. The overall design can achieve a trade-off between the level of measurement noise in the residuals and the ultimate bound on the fault identification error.

The paper is organized as follows. Modeling of robot dynamics with actuation faults is recalled in Sect. II. In Sect. III, the FDI method introduced in [7] for detecting and isolating actuator faults in robot manipulators is briefly summarized. The main result on the identification of the actual profile of the detected actuator faults is presented in Sect. IV. Experimental results are reported in Sect. V for the Quanser Pendubot, a $2 R$ planar robot moving under gravity and having the second joint unactuated.

\section{Modeling}

We consider rigid robot manipulators with $n$ joints that may undergo actuator faults. Using a Lagrangian approach, the standard robot dynamic model is

$$
M(q) \ddot{q}+c(q, \dot{q})+g(q)+F_{v} \dot{q}+F_{c} \operatorname{sign}(\dot{q})=u-u_{f},
$$

where $q$ is he $n$-vector of generalized coordinates, $M(q)>0$ is the (symmetric) inertia matrix, $c(q, \dot{q})$ is the Coriolis and centrifugal vector, $g(q)$ is the gravity vector, $F_{v} \geq 0$ and $F_{c} \geq 0$ are, respectively, the viscous and Coulomb friction (diagonal) matrices, $u$ are the commanded (nominal) torques, and $u_{f}$ are the (unknown) fault torques. 
Note that the right-hand side of eq. (1) captures any type of actuator fault, e.g., a total or partial failure, a torque bias, or a saturation (see [7] for a complete list), with the faults having any time history. In particular, it covers both abrupt and incipient faults.

\section{ACTUATOR FDI SCHEME}

In [7], we have proposed a method for detecting and isolating actuator faults in robotic systems modeled by eq. (1). This is based on the use of the generalized momenta $p=M(q) \dot{q}$. In fact, one can write the following first-order dynamic equation

$$
\dot{p}=u-u_{f}-\alpha(q, \dot{q}),
$$

where, using eq. (1), the components of $\alpha(q, \dot{q})$ are given, for $i=1, \ldots, n$, by

$$
\alpha_{i}=-\frac{1}{2} \dot{q}^{T} \frac{\partial M(q)}{\partial q_{i}} \dot{q}+g_{i}(q)+F_{v i} \dot{q}_{i}+F_{c i} \operatorname{sign}\left(\dot{q}_{i}\right) .
$$

Note that only part of the Coriolis and centrifugal terms $c$ are present in $\alpha$. It is also evident from eq. (2) that each fault (and nominal input torque) affects one and only one component of $p$ and this decoupling allows separate isolation even of concurrent actuator faults, The residual vector is defined as

$$
r=K\left[\int(u-\alpha-r) d t-p\right],
$$

with $K=\operatorname{diag}\left\{k_{1}, \ldots, k_{n}\right\}>0$. Note that the implementation of eq. (4) requires $(q, \dot{q})$ and the nominal input $u$ but no acceleration $\ddot{q}$ nor inversion of the inertia matrix $M(q)$. The residual dynamics satisfies

$$
\dot{r}=-K r+K u_{f}
$$

or

$$
\dot{r}_{i}=-k_{i} r_{i}+k_{i} u_{f i}, \quad i=1, \ldots, n,
$$

namely that of $n$ linear exponentially stable and decoupled systems, each driven by the associated fault input $u_{f i}$.

Remark 1. In principle, by choosing very large gains $k_{i}$ in eq. (5), the behavior of $r_{i}(t)$ would accurately reproduce that of $u_{f i}(t)$. However, this nominal design does not take into account the presence of measurement noise which sets an upper limit to the useful range for the $k_{i}$ 's. Moreover, the presence of uncertainties in the robot dynamic model leads to the introduction of threshold mechanisms in order to prevent false alarms. These thresholds would also become larger for larger values of $k_{i}$, thus reducing fault sensitivity. As a result, arbitrary approximation of the fault profile $u_{f i}(t)$ based only on eq. (5) and a large $k_{i}$ may be an unfeasible option.

\section{FAULT IDENTIFICATION}

Starting from eq. (5), the problem of identifying the evolution of the fault $u_{f i}$ can be formulated as that of observing the state of a linear augmented system driven by the residual $r_{i}$ and by an unknown input. Thanks to the decoupled structure of eq. (5), $n$ independent similar problems can be considered. We assume that the generic fault input $u_{f i}$ is described by a differentiable function with time derivative $\phi_{i}(t)$. Dropping for compactness the index $i$, and having set $x=\left(x_{1}, x_{2}\right)=\left(r, u_{f}\right)$, the generic scalar fault-residual behavior can be described by the two-dimensional dynamic system

$$
\begin{aligned}
\dot{x}_{1} & =-k x_{1}+k x_{2} \\
\dot{x}_{2} & =\phi \\
y & =x_{1},
\end{aligned}
$$

i.e., a linear, time-invariant system in the form

$$
\begin{aligned}
& \dot{x}=A x+B \phi \\
& y=C x,
\end{aligned}
$$

with

$$
A=\left[\begin{array}{rr}
-k & k \\
0 & 0
\end{array}\right], B=\left[\begin{array}{l}
0 \\
1
\end{array}\right], C=\left[\begin{array}{ll}
1 & 0
\end{array}\right] .
$$

Note that the couples $(A, B)$ and $(C, A)$ are controllable and, respectively, observable, since $k \neq 0$.

The time derivative $\phi(t)$ of the fault is an unknown input for system (7). Therefore, in order to identify the actual fault $u_{f}=x_{2}$, one may try to design an observer using the theory for linear systems with unknown inputs [11]. Unfortunately, the necessary conditions for the existence of such observer are violated in this case (in particular, condition $\operatorname{rank}(C B)=\operatorname{rank}(B)$ is not fulfilled). We propose thus an alternative solution that, following the $\mathcal{H}_{\infty}$ paradigm [10], guarantees a uniformly ultimately bounded estimation error, with arbitrarily small ultimate bound [12]. To this aim, we need the following

Assumption. The amplitude of the input signal $\phi(t)$ is bounded by a known constant $\mu>0$, i.e., $|\phi(t)| \leq \mu$.

The observer has the structure

$$
\dot{\xi}=A \xi+F(y-C \xi)
$$

with the $2 \times 1$ matrix $F$ to be determined. Correspondingly, the dynamics of the estimation error $e=x-\xi$ is given by

$$
\dot{e}=(A-F C) e+B \phi .
$$

Note that, whenever matrix $(A-F C)$ is Hurwitz, the following properties are guaranteed for the error $e$ :

- since $\phi(t)$ is bounded, $e(t)$ is also bounded;

- if $\phi(t)$ converges to zero, then also $e(t)$ exponentially converges to zero.

Following [10], it is possible to set a given bound $\varepsilon$ on $\|e\|^{2}$, by choosing matrix $F$ in eq. (9) of the form

$$
F=P^{-1} C^{T} \lambda \text {, }
$$

where the $2 \times 2$ matrix $P$ is symmetric and positive definite, and $\lambda>0$ is a real constant. In fact, consider the positive definite Lyapunov candidate

$$
V=e^{T} P e \geq 0, \quad(V=0 \Leftrightarrow e=0) .
$$

If one is able to establish that, for some admissible $P$ and $\lambda$,

$$
\dot{V} \leq-a V+\mu^{2}
$$


with arbitrary $a>0$, then $V$ is uniformly ultimately bounded (u.u.b.) by

$$
\delta=\frac{\mu^{2}}{a}
$$

with $\delta$ approaching zero as a grows to infinity. Correspondingly, the square norm $\|e\|^{2}$ of the estimation error is u.u.b. by $\varepsilon=\delta / \sigma_{\min }(P)$, where $\sigma_{\min }(P)>0$ is the smallest eigenvalue of matrix $P$. Furthermore, from (13), the exponential convergence of $V$ and thus of $e$ to their ultimate bounds also follow.

The computation of the time derivative of $V$ yields

$$
\begin{aligned}
\dot{V} & =e^{T}\left(P A+A^{T} P-2 C^{T} C \lambda\right) e+2\left(e^{T} P B\right) \phi \\
& \leq e^{T}\left(P A+A^{T} P-2 C^{T} C \lambda+P B B^{T} P\right) e+\mu^{2}
\end{aligned}
$$

where we have used eqs. (10-11), our Assumption, and the triangular inequality. Condition (13) clearly holds if the following matrix inequality is verified

$$
P A+A^{T} P-2 C^{T} C \lambda+P B B^{T} P \leq-a P .
$$

Without loss of generality, the above subproblem can be reformulated as follows. For each $a>0$, find a symmetric, definite positive matrix $P$ and a constant $\lambda>0$ that verify the equation

$$
P A+A^{T} P-2 C^{T} C \lambda+P B B^{T} P=-2 a P,
$$

or

$$
P(A+a I)+(A+a I)^{T} P-2 C^{T} C \lambda+P B B^{T} P=0 .
$$

Since $(A+a I, B)$ is controllable and $(C, A+a I)$ is observable, the Riccati equation (17) is certainly solvable.

For sufficiently large $a$, one possible solution $(P, \lambda)$ is computed as

$$
P_{11}=\frac{3 a^{2}}{2 k^{2}}(3 a-k), \quad P_{12}=P_{21}=-\frac{3 a^{2}}{2 k}, \quad P_{22}=a
$$

and

$$
\lambda=a^{2}\left[\frac{45 a^{2}}{8 k^{2}}-\frac{6 a}{k}+\frac{3}{2}\right]>0,
$$

from which the expression of the observer matrix $F$ in eq. (11) follows.

Remark 2. Since input and measurement noise certainly affect system (1) and thus the residual behavior (6), practical limits exist on the choice of $a$ in eqs. (13-14), and thus on the smallest upper bound that can be obtained for the estimation error $e$. In fact, the value of $a$ reflects in the gain $F$ in eq. (9) and must be chosen as a trade-off between accurate fault tracking and sensitivity to noise.

Remark 3. The level of input noise affecting the residual behaviors (5) can be tuned by suitable choices of the scalar gains in matrix $K$. In particular, a 'small' $K$ should be taken in order to get low sensitivity to noise for the residual generator. The corresponding loss in terms of speed of reaction to faults may then be recovered by the possibility of choosing higher values for parameter $a$ in the observer design. Under the same fault reaction capabilities, the fault estimation $\xi_{2}$ provided by system (9) is often a less noisy signal than the residual $r$ itself. Therefore, $\xi_{2}$ can be used in place of $r$ as a diagnostic signal with higher sensitivity to faults.

\section{EXPERIMENTAL RESULTS}

The proposed fault identification scheme has been experimentally tested on the Quanser Pendubot, a $2 R$ planar robot moving under gravity, with the first joint driven by a DC motor and the second joint passive (see Fig. 1). The dynamic model (1) takes the form

$$
\begin{aligned}
& {\left[\begin{array}{cc}
a_{1}+2 a_{2} c_{2} & a_{3}+a_{2} c_{2} \\
a_{3}+a_{2} c_{2} & a_{3}
\end{array}\right]\left[\begin{array}{c}
\ddot{q}_{1} \\
\ddot{q}_{2}
\end{array}\right]} \\
& +\left[\begin{array}{c}
-a_{2} \dot{q}_{2}\left(\dot{q}_{2}+2 \dot{q}_{1}\right) s_{2} \\
a_{2} \dot{q}_{1}^{2} s_{2}
\end{array}\right]+\left[\begin{array}{c}
a_{4} s_{1}+a_{5} s_{12} \\
a_{5} s_{12}
\end{array}\right] \\
& +\left[\begin{array}{c}
F_{v 1} \dot{q}_{1} \\
F_{v 2} \dot{q}_{2}
\end{array}\right]+\left[\begin{array}{c}
F_{c 1} \operatorname{sign}\left(\dot{q}_{1}\right) \\
F_{c 2} \operatorname{sign}\left(\dot{q}_{2}\right)
\end{array}\right]=\left[\begin{array}{c}
u_{1}-u_{f 1} \\
u_{2}-u_{f 2}
\end{array}\right],
\end{aligned}
$$

where $\left(q_{1}, q_{2}\right)=0$ is the asymptotically stable, free equilibrium configuration and the expressions of the dynamic coefficients $a_{i}$ 's are

$$
\begin{aligned}
& a_{1}=I_{1}+m_{1} d_{1}^{2}+I_{2}+m_{2} d_{2}^{2}+m_{2} l_{1}^{2} \\
& a_{2}=m_{2} d_{2} l_{1} \\
& a_{3}=I_{2}+m_{2} d_{2}^{2} \\
& a_{4}=g\left(m_{1} d_{1}+m_{2} l_{1}\right) \\
& a_{5}=g m_{2} d_{2},
\end{aligned}
$$

being, for the $i$-th link, $m_{i}$ the mass, $l_{i}$ the length, $d_{i}$ the distance of the center of mass from the joint axis, and $I_{i}$ the barycentric inertia, whereas $F_{v i}, F_{c i}$ are the viscous and, respectively, Coulomb friction coefficients for the $i$-th joint ${ }^{1}$.

The evaluation of $\alpha$ from eq. (3) yields in this case

$$
\begin{aligned}
& \alpha_{1}=a_{4} s_{1}+a_{5} s_{12}+F_{v 1} \dot{q}_{1}+F_{c 1} \operatorname{sign}\left(\dot{q}_{1}\right) \\
& \alpha_{2}=a_{2} \dot{q}_{1}\left(\dot{q}_{1}+\dot{q}_{2}\right) s_{2}+a_{5} s_{12}+F_{v 2} \dot{q}_{2}+F_{c 2} \operatorname{sign}\left(\dot{q}_{2}\right) .
\end{aligned}
$$

The joint positions are measured by sliding-contact encoders. On the other hand, joint velocities are obtained by numerical differentiation of positions, which is the main source of measurement noise. The DC motor at the first joint is driven by a PWM circuit, introducing a relevant amount of input noise (and a nonlinear input-output characteristic map).

Several types of faults have been tested on the first joint actuator (bias, saturation, power loss, partial or total actuator failures), while on the second (unactuated) joint only a total actuator failure can be considered when requiring some torque $u_{2}$ that, of course, cannot be provided $\left(u_{f 2} \equiv u_{2}\right)$. Results are reported here for a faulty situation occurring in a feedback control experiment, with the first joint variable being

${ }^{1}$ For the considered robot it is, in particular, $m_{1}=0.193$ and $m_{2}=$ $0.073 \mathrm{Kg}, l_{1}=0.1492$ and $l_{2}=0.1905 \mathrm{~m}, d_{1}=0.1032$ and $d_{2}=0.084 \mathrm{~m}, I_{1}=0.0015$ and $I_{2}=1.949 \cdot 10^{-4} \mathrm{Kgm}^{2}$. Furthermore, $\left[F_{v 1}, F_{v 2}\right]=[5.753,0.281] \cdot 10^{-4} \mathrm{Ns}$, and $\left[F_{c 1}, F_{c 2}\right]=$ $[0.0052,0.0001] \mathrm{Nm}$. 


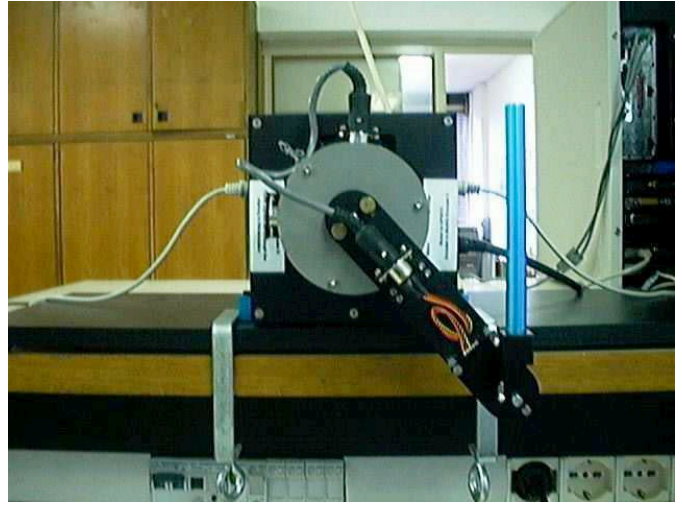

Fig. 1. The Quanser Pendubot used for experiments

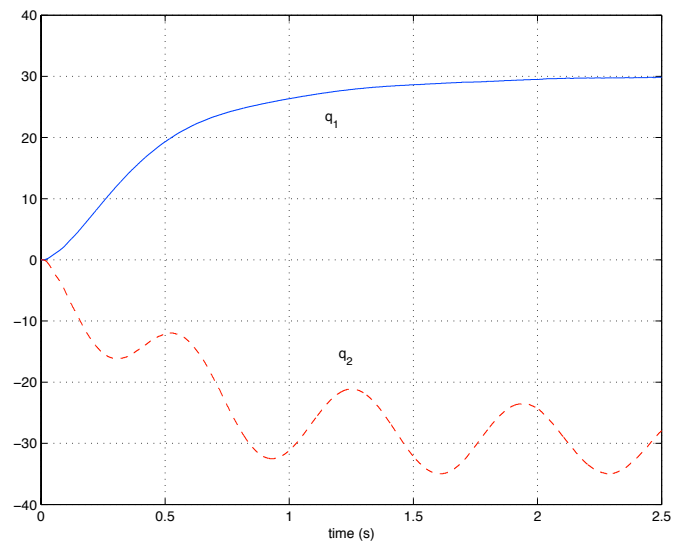

Fig. 2. Joint positions $q_{1}$ (solid, blue) and $q_{2}$ (dashed, red)

regulated to the reference value $q_{1 d}=30^{\circ}$ by a standard PID controller with $K_{P}=0.2, K_{I}=1$, and $K_{D}=0.02$.

A total actuator failure occurs at the second joint for $t \in$ $[0.5,1] \mathrm{s}$ (the commanded torque was $u_{2}=-0.01 \mathrm{Nm}$ ) and a power loss of $10 \%$ affects the actuator of the first joint in the interval $t \in[1.7,2] \mathrm{s}$. The robot joint trajectories and the commanded torques are shown in Fig. 2 and, respectively, in Fig. 3. Note that the considered faults are of low severity and thus are not easily detectable from the measured position profiles or from the torque history.

The use of the FDI method (4) alone, with large gains but without an explicit identification scheme, is evaluated first. The residuals of Fig. 4 were obtained using $k_{1}=k_{2}=50$. Reaction to faults is very fast, but the fault event at the first joint is not clearly detectable due to the high level of noise affecting the associated residual. As a matter of fact, both residuals exceed several times the chosen thresholds for fault detection even during normal operation (false alarms). On the other hand, larger thresholds may fail to detect the fault on actuator 1 (see Fig. 4). Moreover, low-pass filtering of the residuals in order to eliminate noise would slow down the response time of the detection and identification scheme.

Next, the complete detection and identification scheme has been implemented using lower gains $k_{1}=k_{2}=0.01$ in eq. (4) and selecting a value $a=10$ in eqs. (18-19), which
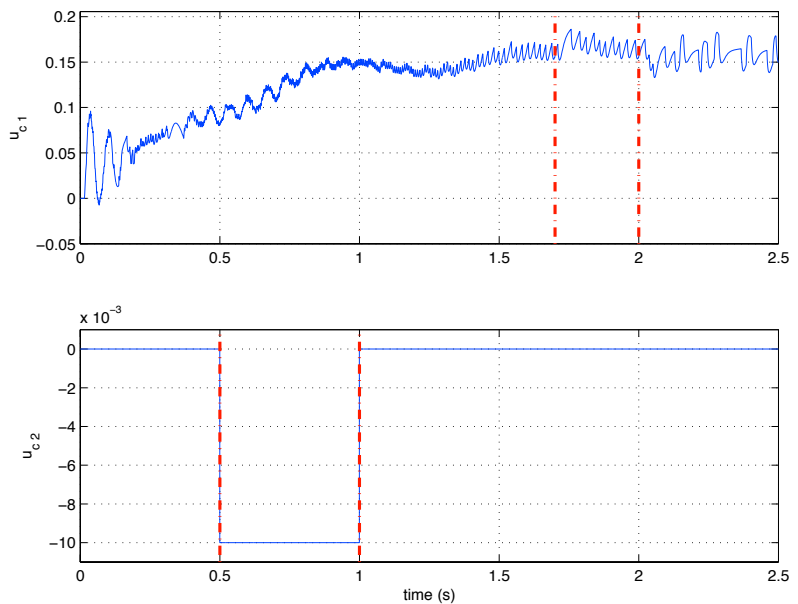

Fig. 3. Commanded torques $u_{1}$ (top) and $u_{2}$ (bottom) - fault intervals are also indicated
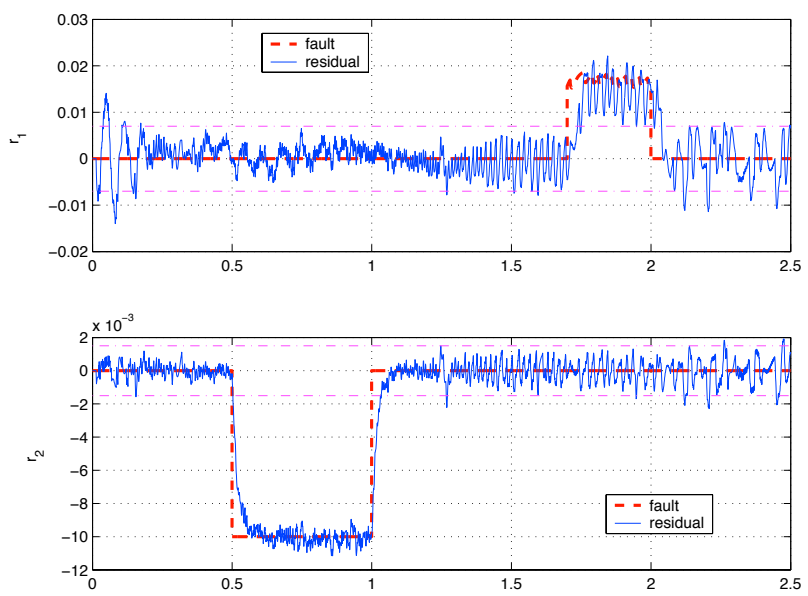

Fig. 4. Residuals $r_{1}$ and $r_{2}$ (solid, blue) for $k_{1}=k_{2}=50$ and actuator faults (dashed, red) - detection thresholds are also indicated

results in an observer gain matrix

$$
F=\left[\begin{array}{ll}
24.99 & 37485
\end{array}\right]^{T} .
$$

Figure 5 shows the behavior of the residuals $r_{1}$ and $r_{2}$. The choice of very low values for $k_{1}$ and $k_{2}$ leads to low levels of noise affecting these signals. As expected, reaction to faults is quite slow, so that a relevant delay would characterize the detection of fault start/end when using these signals for diagnosis. However, the estimated fault behaviors reported in Fig. 6 show good tracking capabilities despite of the relevant noise and disturbances affecting the system. Since a bound on the derivative of the fault can be been computed as $\mu=0.2$ and $\sigma_{\min }(P)=4.9983$, the theoretical ultimate bound on the squared norm of the estimation error is $\varepsilon \simeq 8 \cdot 10^{-4}$, which turns out to be quite conservative in the present case.

It is worth noting that the estimated fault torques in Fig. 6 are more suitable diagnostic signals than the residuals in Fig. 4, which were obtained with higher gains. In particular, 

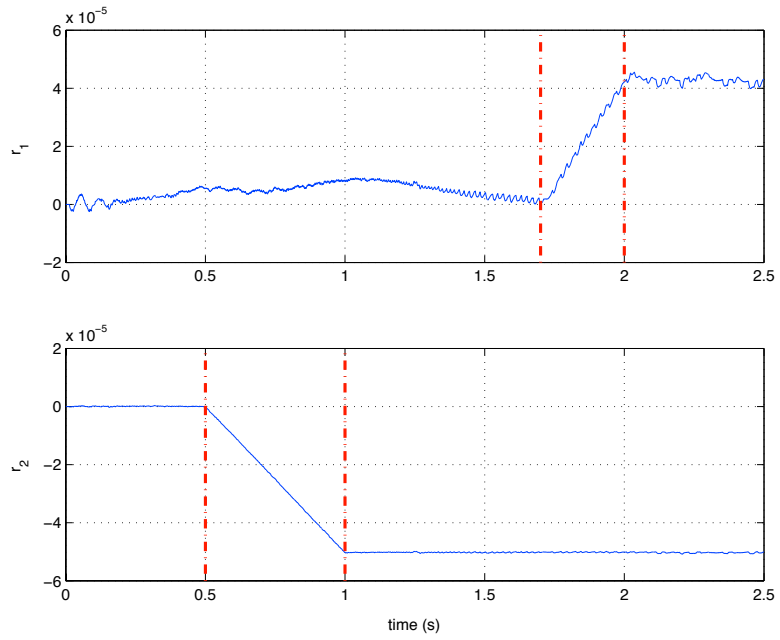

Fig. 5. Residuals $r_{1}$ and $r_{2}$ for $k_{1}=k_{2}=0.01-$ fault intervals are also indicated
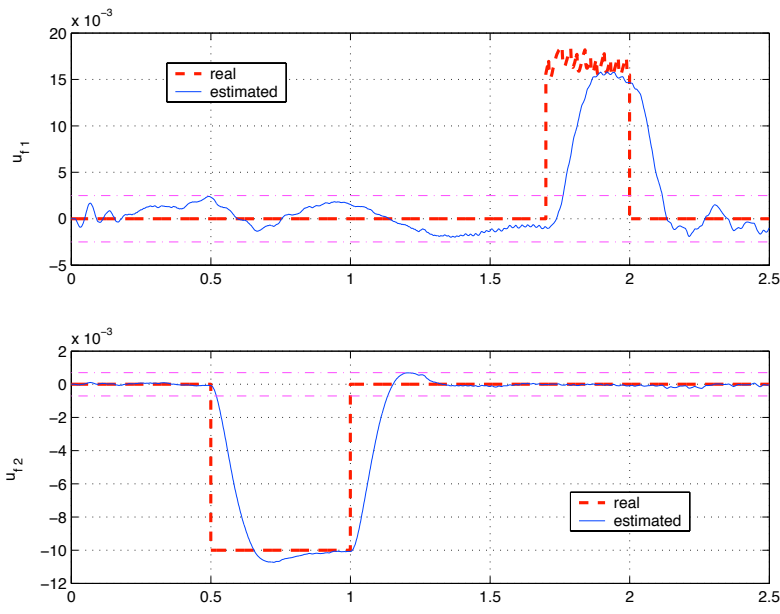

Fig. 6. Actuator faults: real (dashed, red) and estimated (solid, blue) for $k_{1}=k_{2}=0.01$ and $a=10-$ detection thresholds are also indicated

the occurrence and end of the faults are clearly and promptly detected (and isolated). To this purpose, much lower threshold values can now be taken (compare these in Fig. 4 and Fig. 6), getting thus better fault sensitivity and no false alarms. Finally, the fault severity can be evaluated by comparing the identified fault behaviors with the commanded joint torques displayed in Fig. 3.

\section{CONCLUSIONS}

Faults of general type affecting the actuators of a robot manipulator can be detected, isolated, and identified though the combined use of generalized momenta of the robot dynamics and a linear robust observer of fault time profiles, allowing an estimation error that is ultimately bound by an arbitrarily small value.

The implementation of these concepts on a robot with uncertain dynamics (mostly due to friction and actuator nonlinearities) and with only joint position measures available (with associated need of numerical differentiation for joint velocity evaluation) has shown the relevant role played by input and measurement noise on the fault detection and identification problems. The presented approach allows the independent tuning of only two detection and identification gains for each actuator channel, so as to obtain satisfactory diagnostic signals in the presence of realistic levels of uncertainty and noise. Further improvements can be expected from an adaptation mechanism that copes with parametric model uncertainties (see [8]) and from an explicit modeling and quantitative characterization of disturbances and noise covariances, within an optimal robust observer approach.

The presented scheme works independently of the control scheme used to generate the torque input applied to the robot. Indeed, the collected information on the identified actuator fault should be used in different ways for control reconfiguration, depending on the specific feedback law in action. For instance, the reported results indicate that a mild power loss of the actuator is well tolerated in terms of performance degradation when using a PID control law for joint-space regulation.

\section{ACKNOWLEDGMENT}

We thank Prof. Stefano Battilotti for useful discussions.

\section{REFERENCES}

[1] M. Visinsky, J. Cavallaro, and I. Walker, "A dynamic fault tolerance framework for remote robots," IEEE Trans. on Robotics and Automation, vol. 11, no. 4, pp. 477-490, 1995.

[2] S. Simani, C. Fantuzzi, and R. Patton, Model-based Fault Diagnosis in Dynamic Systems using Identification Techniques. London: Springer, 2002.

[3] H. Schneider and P. Frank, "Observer-based supervision and fault detection in robots using nonlinear and fuzzy logic residual evaluation," IEEE Trans. on Control Systems Technology, vol. 4, no. 3, pp. 274-282, 1996.

[4] A. Vemuri and M. Polycarpou, "Neural-network-based robust fault diagnosis in robotic systems," IEEE Trans. on Neural Networks, vol. 8, no. 6, pp. 1410-1420, 1997.

[5] F. Caccavale and L. Villani, "Fault diagnosis for industrial robots," in Fault Diagnosis and Fault Tolerance for Mechatronic Systems: Recent Advances (F. Caccavale and L. Villani, eds.), vol. 1 of STAR, pp. 85108, Springer, 2003.

[6] W. Dixon, I. Walker, D. Dawson, and J. Hartranft, "Fault detection for robot manipulators with parametric uncertainty: A prediction-errorbased approach," IEEE Trans. on Robotics and Automation, vol. 16, no. 6, pp. 689-699, 2000.

[7] A. De Luca and R. Mattone, "Actuator fault detection and isolation using generalized momenta," in Proc. of 2003 IEEE Int. Conf. on Robotics and Automation, (Taipei, Taiwan), pp. 634-639, 2003.

[8] A. De Luca and R. Mattone, "An adapt-and-detect FDI system for robot manipulators," in Proc. of 2004 IEEE Int. Conf. on Robotics and Automation, (New Orleans, LA), pp. 4975-4980, 2004.

[9] M. McIntyre, W. Dixon, D. Dawson, and I. Walker, "Fault detection and identification for robot manipulators," in Proc. of 2004 IEEE Int. Conf. on Robotics and Automation, (New Orleans, LA), pp. 4981-4986, 2004.

[10] J. Doyle, K. Glover, P. Khargonekar, and B. Francis, "State-space solutions to standard $\mathcal{H}_{2}$ and $\mathcal{H}_{\infty}$ control problems," IEEE Trans. on Automatic Control, vol. 34, no. 8, pp. 831-847, 1989.

[11] Y. Guan and M. Saif, "A novel approach to the design of unknown input observers," IEEE Trans. on Automatic Control, vol. 36, no. 5, pp. 632-635, 1991.

[12] H. Khalil, Nonlinear Systems. New York: Macmillan, 1992. 